\title{
Analysis of the profile roughness of core-shell microparticles by electron microscopy
}

\author{
Deniz Hülagü ${ }^{1}$, Charlie Tobias ${ }^{2}$, Ardian Gojani $^{3}$, Knut Rurack ${ }^{2}$ and Vasile-Dan Hodoroaba ${ }^{1}$
}

${ }^{1}$ Federal Institute for Materials Testing and Research (BAM), Division 6.1 Surface Analysis and Interfacial Chemistry, Berlin, Berlin, Germany, ${ }^{2}$ Federal Institute for Materials Testing and Research (BAM), Division 1.9 Chemical and Optical Sensing, Berlin, Berlin, Germany, ${ }^{3}$ Federal Institute for Materials Testing and Research (BAM), Division 1.9 Chemical and Optical Sensing, Berlin, Germany

Bead-based immunoassays play an increasingly important role in various fields of the analytical sciences. In particular, few micron-sized beads are well suited for the quantitative detection of analytes of interest with their rapid reaction kinetics and requirement of small sample volumes. A very promising material combination for bead-based immunoassays is achieved by merging the benefits of polystyrene (PS) and silica $\left(\mathrm{SiO}_{2}\right)$ particles in a core-shell (CS) particle format $[1,2]$. Besides the particle synthesis procedure, characterization is also essential to establish reproducibility and the performance of the particles [3]. Particle characterization involving the measurements of size, size distribution, shell thickness and surface texture, surface area and morphology has been commonly conducted with different analytical methods [4]. The mechanical and wetting properties of the CS particles are significantly influenced by their roughness $[3,5,6]$. However, the literature lacks a systematic evaluation of the roughness of the single particles. This work provides a reliable tool to calculate a particle's profile roughness with high accuracy and reproducibility.

The particles used in this study were prepared in the frame of the Focus Area project of BAM "MamaLoCA Modular, multiplexed, antibody-based lab-on-chip analyzer for food control [7]". The following samples have been investigated: i) PS particles synthesized by dispersion polymerization [1], ii) PS particles covered with an iron oxide $\left(\mathrm{Fe}_{3} \mathrm{O}_{4}\right)$ layer and iii) CS particles with a PS core covered with a $\mathrm{Fe}_{3} \mathrm{O}_{4}$ layer and closed with a second silica $\left(\mathrm{SiO}_{2}\right)$ shell. The particles have been suspended in ethanol and samples for analysis have been prepared by drop-casting on conventional carbon TEM grids. Images of single particles were taken by a dual mode Scanning Electron Microscopy (SEM) set-up which allows imaging of the same field-of-view of the sample with high-resolution and surface sensitive in SE InLens ${ }^{\circledR}$ mode as well as in transmission mode (TSEM).

A particle roughness analysis tool, based on electron microscopy (EM) images, was previously reported by Sarma et al [3]. In the present work, a new Python script was developed to evaluate quantitatively EM images with respect to their surface roughness. The steps of image analysis are depicted in Figure 1 for a SEM image of a single particle (a). First, an automated threshold based on IsoData algorithm was applied to the image with the Gwyddion software (http://gwyddion.net/) to obtain a binary image (b). The gross errors which might affect the accuracy of the image segmentation were manually corrected (c). The rest is taken over by the developed Python script. The script scans each pixel of the binary image and identifies all possible boundary points when the pixel changes from black to white, or vice versa (d). Hence, the complete contour of the particle is identified. The initial center point of the particle is calculated as the average of the identified border points (e). The optimized center point is calculated by minimizing the standard deviation (SD) of the distances between the center point and each border point (f). Finally, the profile roughness of the particle is calculated as the SD of the distances. The same procedure is applied to the TSEM images.

Figure 2 presents the comparison of the radius and profile roughness of a single $\mathrm{PS} / \mathrm{Fe}_{3} \mathrm{O}_{4} / \mathrm{SiO}_{2}$ particle calculated with the developed procedure using SEM and TSEM images of the same particle. The difference between the calculated mean radii was negligible (1.2\%). However, the SEM-based analysis resulted in a significantly rougher surface with a (13.4\%) higher SD than that of TSEM-based analysis. This is probably attributed to the saturation of the InLens ${ }^{\circledR}$ signal at the particle boundary. Another likely reason is the difference in the grey value of the images, correspondingly, different threshold settings. Because of the higher threshold 
applied to the TSEM-based analysis the fine features of the particles are not clearly identified, which resulted in lower roughness.

The influence of various parameters on the calculated roughness was also investigated: the setting of the proper threshold, accelerating voltage, etc. The samples were gradually tilted to extend imaging information of more than only one projection. Furthermore, the measurement uncertainty of the profile roughness of particles associated to various orientations was estimated.

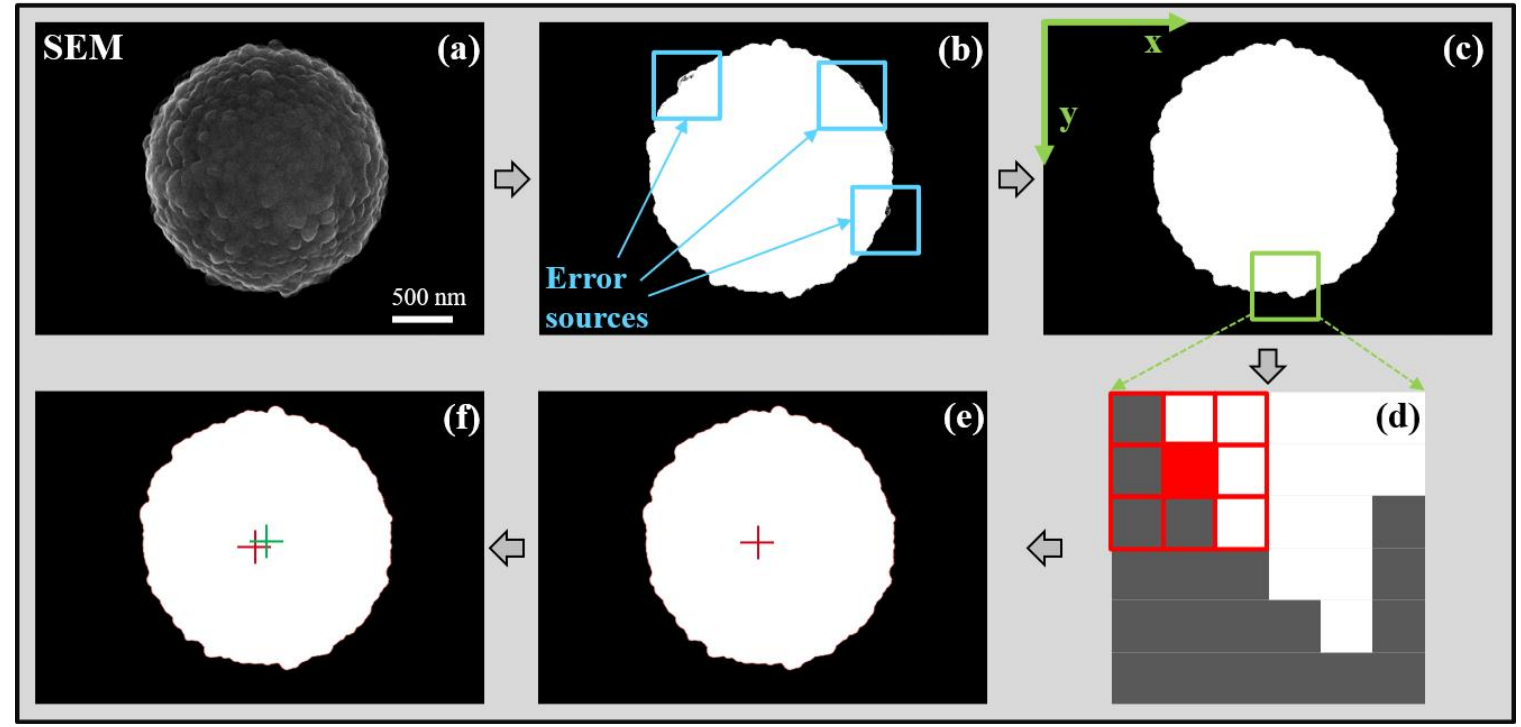

Figure 1. Schematic illustration of processing sequence of a SEM/InLens image (a). The binary image obtained by automated threshold with Gwyddion (b), which was next manually corrected (c). Identification of the boundary points (d), calculated initial center (e), and optimized center (f) with the custom Python script.

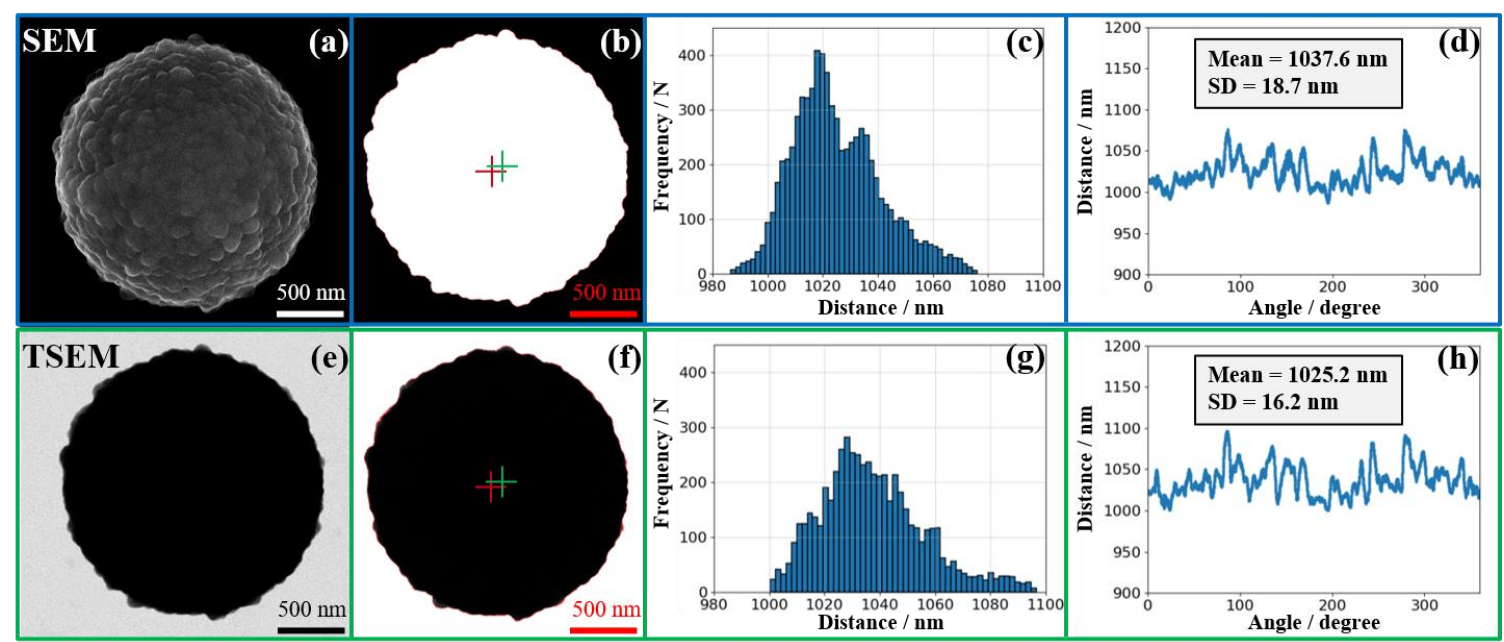

Figure 2. A single particle from SEM (a) and TSEM (e) micrographs are compared after being transformed into binary images ( $b$ and $f$ ). The calculated distances from the optimized center point (c and $\mathrm{g}$ ) are plotted as a function of angle ( $\mathrm{d}$ and $\mathrm{h}$ ). Then the radius and the roughness are calculated as mean and standard deviation (SD) of the distances, respectively. 


\section{References}

[1] C. Tobias et al., ACS Appl. Mater. Interfaces, 2020 13(1) p. 207-218.

[2] D. Sarma et al., Langmuir, 2016 32(15) p. 3717-3727.

[3] D. Sarma et al., Appl. Surf. Sci., 2017426 p. 446-455.

[4] M. M. Modena et al., Adv. Mater., 2019 31(32) p. 1901556.

[5] X. Li and and J. He, CS Appl. Mater. Interfaces, 2012 4(4) p. 2204-2211.

[6] L. Mammen et al., Langmuir, 2012 28(42) p. 15005-15014.

[7] https://www.bam.de/Content/EN/Projects/MamaLoCA/mamaloca.html, 2021. 